Author affiliations and support information (if applicable) appear at the end of this article.

Published at jco.org on May 25, 2017.

Corresponding author: Renée M.F. Ebisch, MD, Department of Obstetrics \& Gynaecology, Radboud University Medical Center, PO Box 9101, 6500 HB Nijmegen, the Netherlands; e-mail: renee. ebisch@radboudumc.nl.

() 2017 by American Society of Clinical Oncology

$0732-183 X / 17 / 3522 w-2542 w / \$ 20.00$

\title{
Long-Lasting Increased Risk of Human Papillomavirus-Related Carcinomas and Premalignancies After Cervical Intraepithelial Neoplasia Grade 3: A Population-Based Cohort Study
}

Renée M.F. Ebisch, Dominiek W.E. Rutten, Joanna IntHout, Willem J.G. Melchers, Leon F.A.G. Massuger, Johan Bulten, Ruud L.M. Bekkers, and Albert G. Siebers

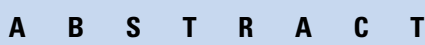

\section{Purpose}

The aim of this study was to determine the risk of human papillomavirus (HPV)-related carcinomas and premalignancies in women diagnosed with cervical intraepithelial neoplasia grade 3 (CIN3). Knowledge of this risk is important to preventing the development and progression of other HPVrelated premalignancies and carcinomas, by considering prophylactic HPV vaccination and/or by paying increased attention to other HPV-related carcinomas and premalignancies when CIN3 is identified.

\section{Methods}

Women diagnosed with a CIN3 between 1990 and 2010 were identified from the Dutch nationwide registry of histopathology and cytopathology (PALGA) and matched with a control group of women without CIN3. Subsequently, all cases of high-risk (hr) HPV-associated high-grade lesions and carcinomas in the anogenital region and oropharynx between 1990 and 2015 were extracted. Incidence rate ratios were estimated for carcinomas and premalignancies of the vulva, vagina, anus, and oropharynx.

\section{Results}

A total of 178,036 women were identified: 89,018 with a previous diagnosis of CIN3 and 89,018 matched control subjects without a history of CIN3. Women with a history of CIN3 showed increased risk of HPV-related carcinomas and premalignancies, with incidence rate ratios of 3.85 (95\% $\mathrm{Cl}, 2.32$ to 6.37$)$ for anal cancer, $6.68(95 \% \mathrm{Cl}, 3.64$ to 12.25$)$ for anal intraepithelial neoplasia grade 3 , 4.97 (95\% Cl, 3.26 to 7.57) for vulvar cancer, 13.66 (93\% Cl, 9.69 to 19.25) for vulvar intraepithelial neoplasia grade 3, $86.08(95 \% \mathrm{Cl}, 11.98$ to 618.08) for vaginal cancer, 25.65 (95\% Cl, 10.50 to 62.69) for vaginal intraepithelial neoplasia grade 3, and $5.51(95 \% \mathrm{Cl}, 1.22$ to 24.84$)$ for oropharyngeal cancer. This risk remained significantly increased, even after long-term follow-up of up to 20 years.

\section{Conclusion}

This population-based study shows a long-lasting increased risk for HPV-related carcinomas and premalignancies of the anogenital and oropharyngeal region after a CIN3 diagnosis. Studies that investigate methods to prevent this increased risk in this group of patients, such as intensified screening or vaccination, are warranted.

\section{J Clin Oncol 35:2542-2550. (C) 2017 by American Society of Clinical Oncology}

\section{INTRODUCTION}

Infections with a high-risk human papillomavirus (hrHPV) are estimated to be the cause of $5.2 \%$ of all cancers worldwide. ${ }^{1}$ HrHPV infections in women cause cervical cancer and cervical intraepithelial neoplasia (CIN), as well as carcinomas and premalignancies of the oropharynx and anogenital region including the vulva, vagina, and anus.

The prevalence of hrHPV in cervical cancer is close to $100 \%$. In vaginal cancers, the prevalence of hrHPV is $60 \%$ to $70 \%$, and in vulvar cancer it ranges from $20 \%$ to $50 \%$. In anal cancer, an hrHPV infection is found in $88 \%$ to $95 \%$ of all cases, and $15 \%$ to $65 \%$ of oropharyngeal cancers are related to the $\mathrm{HPV}^{2,3}$ 
It has been shown that women with a diagnosis of CIN grade 3 (CIN3) are at increased risk of carcinomas of the anogenital and head and neck region, probably as the result of an infection with an hrHPV. ${ }^{4-8}$ However, none of those studies included the risk for high-grade premalignancies after a diagnosis of CIN3 ${ }^{4,5,8-10}$ Knowledge of this risk is important to preventing the development and progression of other HPV-related premalignancies and carcinomas, by considering prophylactic HPV vaccination and/or by paying increased attention to other HPV-related carcinomas and premalignancies when CIN3 identified. Primary prevention of HPV-related carcinomas and premalignancies by prophylactic vaccination is now only available for young women. Older and unvaccinated women are therefore still at risk for HPV-related disease in the next decades. Treating premalignancies in a cohort may prevent malignancies from developing, and the risk for HPVrelated carcinomas and premalignancies combined represents the possible preventive effect of HPV vaccination in women with a CIN3. The aim of this study was therefore to determine the risk of HPV-related carcinomas and premalignancies in women with a previous histologic diagnosis of CIN3.

\section{METHODS}

Women with a diagnosis of CIN3 between January 1, 1990, and December 31,2010 , were identified from the Dutch nationwide registry of histopathology and cytopathology (PALGA; Houten, Netherlands). Date of birth and first four letters of the surname were used as a personal identifier. Women registered in the database with a benign dermal nevus, but who were never diagnosed with CIN3 or cervical carcinoma before or after the diagnosis of nevus, were selected from the database as a control group. Registration of a pathology result in the database was needed to obtain a control group from the database; a registered benign dermal nevus was chosen because it is not related to HPV and can be diagnosed in all women. Women with both a CIN3 and a benign nevus diagnosis were included in the CIN3 group. Control subjects were assigned a random number and sorted. Subsequently, from the total group of 547,924 matched women with a nevus, random frequency matching was done by age and year of detection (both within a range of 5 years), and population density areas; smaller cities with $<100,000$ inhabitants were considered low-density areas, and those with $>100,000$ inhabitants were considered higherdensity cities. For all identified women, histology results for carcinomas and high-grade premalignancies of the vulva, vagina, perineum, anus, and oropharynx between January 1, 1990, and March 1, 2015, were retrieved, resulting in 4 to 25 years of follow-up. In addition, patient age and date of diagnosis were retrieved.

We identified all HPV-related carcinomas and premalignancies diagnosed after the CIN3 or benign dermal nevus. Only results with a clear diagnosis of malignancy were classified as cancer; when invasiveness was uncertain in a high-grade lesion, the result was classified as a high-grade premalignancy. Moderate (CIN2) and low-grade (CIN1) premalignancies were not included in the high-grade group. Women were censored after diagnosis of an HPV-related carcinoma of any site because treatment might influence the subsequent risk of other carcinomas or premalignancies. Women with an HPV-related carcinoma or premalignancy before the CIN3 or benign dermal nevus were excluded from analysis, as well as results indicating recurrence or metastases of disease. Differentiated vulvar intraepithelial neoplasia (VIN) and vulvar cancer after a previous diagnosis of lichen sclerosis, clear cell carcinomas, serous adenocarcinoma, lymphomas, melanomas, and basal cell carcinomas were also excluded because these are not likely to be related to HPV. ${ }^{11,12}$

We calculated person-years at risk and the number of observed carcinomas and premalignancies in each group, taking into account censoring as described in the previous paragraph. Using Poisson regression with an offset on the basis of the person-years, incidence rates (IR) per 100,000 person-years were estimated for both cases and their matched controls. Incidence rate ratios (IRR), comparing women with a CIN3 diagnosis with the matched control group, were computed, first by analyzing the whole follow-up period and second by excluding the first year of follow-up, to account for possibly prevalent HPVrelated carcinomas and premalignancies in the cohort. HPV-related carcinomas and premalignancies were analyzed separately and combined into clusters of any HPV-related carcinoma and/or any HPVrelated premalignancy. The cluster of any HPV-related carcinoma includes anal cancer, vulvar cancer, vaginal cancer, and oropharyngeal cancer. The cluster of any HPV-related premalignancy includes anal intraepithelial neoplasia grade 3 (AIN3), VIN grade 3 (VIN3), and vaginal intraepithelial neoplasia grade 3 (VAIN3). The cluster of any malignancy or premalignancy includes anal cancer, AIN3, vulvar cancer, VIN3, vaginal cancer, VAIN3, and oropharyngeal cancer. In all analyses, person-years were only considered before the first diagnosed carcinoma or premalignancy.

Age-adjusted IRRs for 5-year follow-up intervals were estimated for up to 25 years of follow-up for each carcinoma and premalignancy separately and for the clustered carcinomas and premalignancies, by means of a Poisson model with an interaction term for the group with a history of CIN3 and follow-up period and a continuous variable for age. In addition, IRRs were estimated for women $\leq 29$ years, 30 to 49 years, 50 to 69 years, and $\geq 70$ years of age for the clustered carcinomas and premalignancies. Kaplan-Meier curves were used to visualize the risk of HPV-related carcinomas and premalignancies over time.

All statistical analyses were performed with SAS version 9.2 (SAS Institute, Cary, NC). Kaplan-Meier curves were obtained with SPSS version 22.0.0.1 for Windows (SPSS, Chicago, IL).

The study was approved by the scientific committee of PALGA. The study was exempt from institutional review board approval because data were gathered retrospectively and analyzed anonymously.

\section{RESULTS}

We identified 178,036 women, of whom 89,018 had a histologic diagnosis of CIN3, between 1990 and 2010, and an equal number of women in the matched control group of women without a history of CIN3. The median age at diagnosis was 35 years in women with a previous diagnosis of CIN3, and 36 years in the control group. Characteristics of the study population at inclusion are listed in Table 1. During a median follow-up of 14 years in both groups (range, 4 to 25 years), 1,261,804 person-years were accrued in the group with a previous diagnosis of CIN3. After censoring, a total of 299 HPV-related carcinomas and 634 HPV-related premalignancies were found in the group with a previous diagnosis of CIN3. In the control group, 1,262,998 person-years were accrued, with $48 \mathrm{HPV}$-related carcinomas and $50 \mathrm{HPV}$-related premalignancies (Table 2).

The risk of developing HPV-related carcinomas and premalignancies of the anus, vulva, vagina, and oropharynx is strongly associated with a previous diagnosis of CIN3, with a significant increase in the group with a history of CIN3 (Fig 1). The IRRs for anal cancer and AIN3, comparing women with a previous diagnosis of CIN3 with those without a previous diagnosis of CIN3, were 3.85 (95\% CI, 2.32 to 6.37 ) and 6.68 (95\% CI, 3.64 to 12.25 ), respectively. For vulvar cancer and VIN3, the IRRs were 4.97 (95\% CI, 3.26 to 7.57 ) and 13.66 (95\% CI, 9.69 to 19.25 ), respectively. Vaginal cancer and VAIN3 showed the highest IRRs, with 86.08 
Table 1. Characteristics of Study Population at Inclusion

\begin{tabular}{|c|c|c|c|c|}
\hline \multirow[b]{2}{*}{ Characteristic } & \multirow[b]{2}{*}{ No. of Patients } & \multirow[b]{2}{*}{ Percentage of Patients } & \multicolumn{2}{|c|}{ Person-Years of Follow-Up } \\
\hline & & & CIN3 History & No CIN3 History \\
\hline \multicolumn{5}{|l|}{ Age cohort, years } \\
\hline$<20$ & 143 & 0.2 & 1,894 & 1,905 \\
\hline $20-24$ & 2,160 & 2.4 & 32,625 & 32,722 \\
\hline $25-29$ & 10,523 & 11.8 & 161,919 & 161,814 \\
\hline $30-34$ & 25,426 & 28.6 & 350,931 & 351,628 \\
\hline $35-39$ & 20,828 & 23.4 & 307,335 & 306,170 \\
\hline $40-44$ & 13,390 & 15.0 & 185,361 & 186,021 \\
\hline $45-49$ & 7,749 & 8.7 & 104,492 & 105,295 \\
\hline $50-54$ & 3,896 & 4.4 & 54,638 & 54,461 \\
\hline $55-59$ & 2,156 & 2.4 & 28,199 & 28,272 \\
\hline $60-64$ & 1,257 & 1.4 & 16,642 & 16,703 \\
\hline $65-69$ & 505 & 0.6 & 7,602 & 7,683 \\
\hline $70-74$ & 418 & 0.5 & 5,540 & 5,570 \\
\hline $75-79$ & 303 & 0.3 & 3,113 & 3,220 \\
\hline$\geq 80$ & 264 & 0.3 & 1,511 & 1,535 \\
\hline Total & 89,018 & 100 & $1,261,804$ & $1,262,998$ \\
\hline \multicolumn{5}{|c|}{ Calendar years of inclusion } \\
\hline 1990-1994 & 20,354 & 22.9 & 458,469 & 457,284 \\
\hline 1995-1999 & 20,785 & 23.3 & 364,119 & 365,151 \\
\hline $2000-2004$ & 19,063 & 21.4 & 239,718 & 239,056 \\
\hline $2005-2010$ & 28,816 & 32.4 & 199,498 & 201,507 \\
\hline Total & 89,018 & 100 & $1,261,804$ & $1,262,998$ \\
\hline
\end{tabular}

Abbreviation: CIN3, cervical intraepithelial neoplasia grade 3 .

NOTE. The number of patients and percentages of age distribution and calendar years of inclusion are similar in both groups because of 5 -year matching

(95\% CI, 11.99 to 618.08 ) for vaginal cancer and 25.65 (95\% CI, 10.50 to 62.69$)$ for VAIN3, as the result of low IRs in the control group. Oropharyngeal cancer showed an IRR of 5.51 (95\% CI, 1.22 to 24.84$)$. The IRR for any HPV-related carcinoma was $6.24(95 \%$ CI, 4.60 to 8.46 ), for any HPV-related premalignancy the IRR was 12.75 (95\% CI, 9.56 to 17.00), and for any HPV-related carcinoma or premalignancy the IRR was 9.68 (95\% CI, 7.77 to 12.05). Excluding the first year after inclusion resulted in IRR estimates that were not notably different (Table 2).

The age-adjusted IRR stratified by time interval since first diagnosis showed a short-term and a long-lasting increased risk of developing HPV-related carcinomas and premalignancies in women with a previous diagnosis of CIN3, when compared with the control group of women without a CIN3 diagnosis. Even up to 20 years after the CIN3 diagnosis, this risk remained increased (Table 3). The increased cumulative incidence over time is clearly visualized in the Kaplan-Meier curves (Fig 2). For the cluster of any HPV-related carcinoma or premalignancy, the IRR decreased over time; however, an increased risk was still visible after long-term follow-up (Fig 3).

IRR stratified by attained age showed that the risk of carcinoma or premalignancies for women with a history of CIN3 might be dependent on age. The IRR of any HPV-related premalignancy and of any carcinoma or premalignancy was highest in women $\leq$ 29 years of age, both with an IRR $>30$. The IRR for women with any HPV-related carcinoma was not available. In the group of women between 30 and 49 years of age, the risk was lower compared with women $\leq 29$ years of age; however, the risk was higher compared with older age groups, with IRRs of 8.09 (95\% CI, 4.81 to 13.58$)$ for any HPV-related carcinoma, 16.68 (95\% CI, 11.14 to 24.96 ) for any HPV-related premalignancy, and 13.62
(95\% CI, 9.76 to 19.01) for any HPV-related carcinoma or premalignancy (Appendix Table A1, online only).

\section{DISCUSSION}

This population-based cohort study, which included 178,036 women, showed an increased risk of premalignancies as well as carcinomas of the anus, vulva, vagina, and oropharynx in women with a previous diagnosis of CIN3 compared with women without a previous diagnosis of CIN3. The increased risk was still present up to 20 years after the CIN3 diagnosis.

Our results are consistent with published studies on HPVrelated carcinomas in women with a CIN diagnosis, with risk ratios ranging from 2.2 to 4.4 for vulvar cancer, 6.7 to 18.5 for vaginal cancer, 1.8 to 5.9 for anal cancer, and 1.2 for oral/pharyngeal tumors. ${ }^{4,5,7-9}$ No studies have analyzed the risk of HPV-related high-grade premalignancies. In general, high-grade premalignancies are treated before cancer can occur. Our data on IRs of high-grade premalignancies showed high IRRs, indicating that in the group of women with CIN3, carcinomas may have been prevented by early detection and treatment of high-grade premalignancies. Our data overall showed higher IRRs compared with published results. This might be caused by differences in our study design compared with published studies. Some studies used expected IRs on the basis of national averaged IRs, which may also include carcinomas in women with a CIN3 in the control group, whereas others used relative risks. Furthermore, the studies by Kalliala et $\mathrm{al}^{4}$ and Gaudet et $\mathrm{al}^{8}$ included women with CIN1 and/or CIN2, of which CIN1 is known to show a lower prevalence of hrHPV compared with CIN2/3. ${ }^{13}$ 


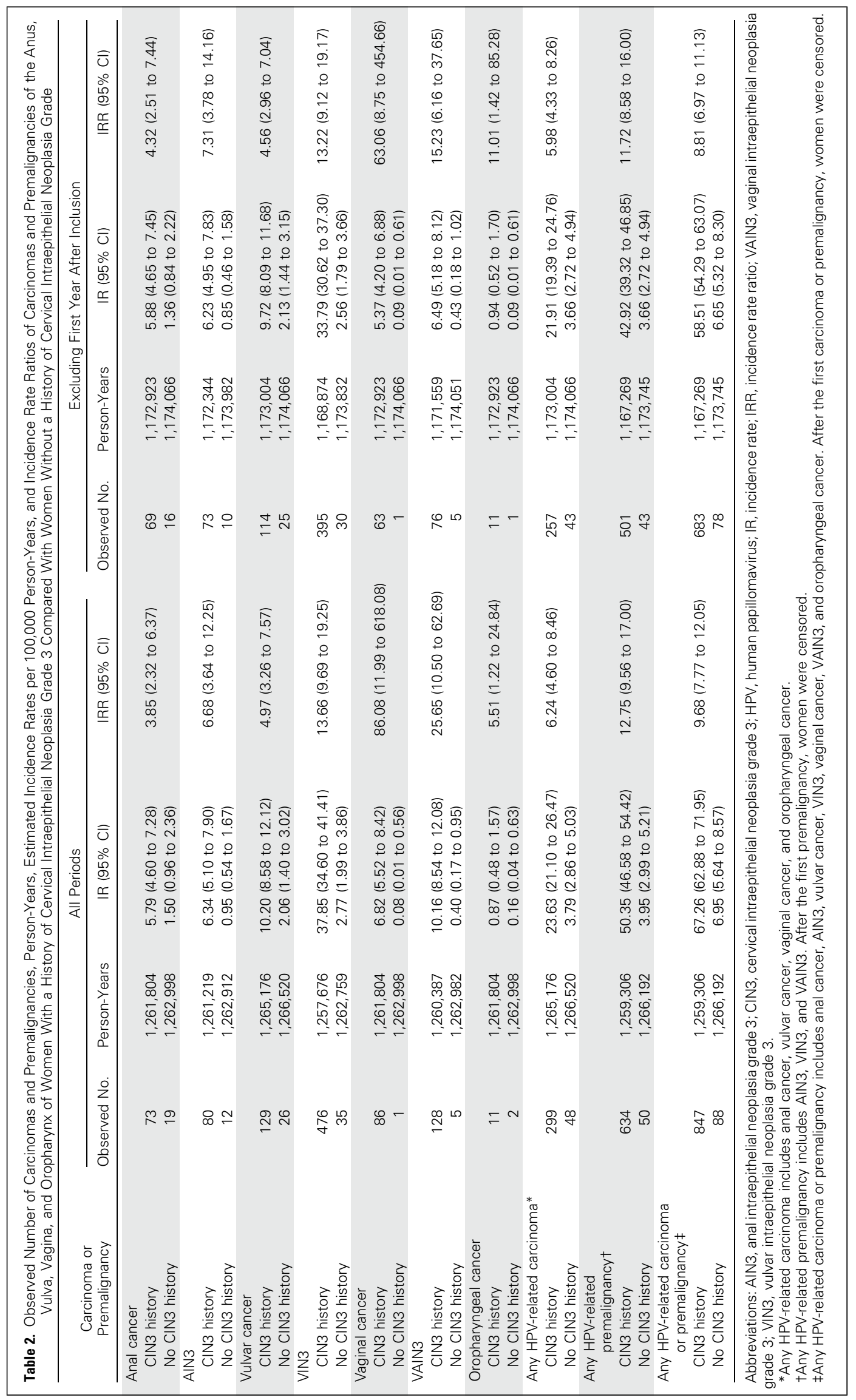




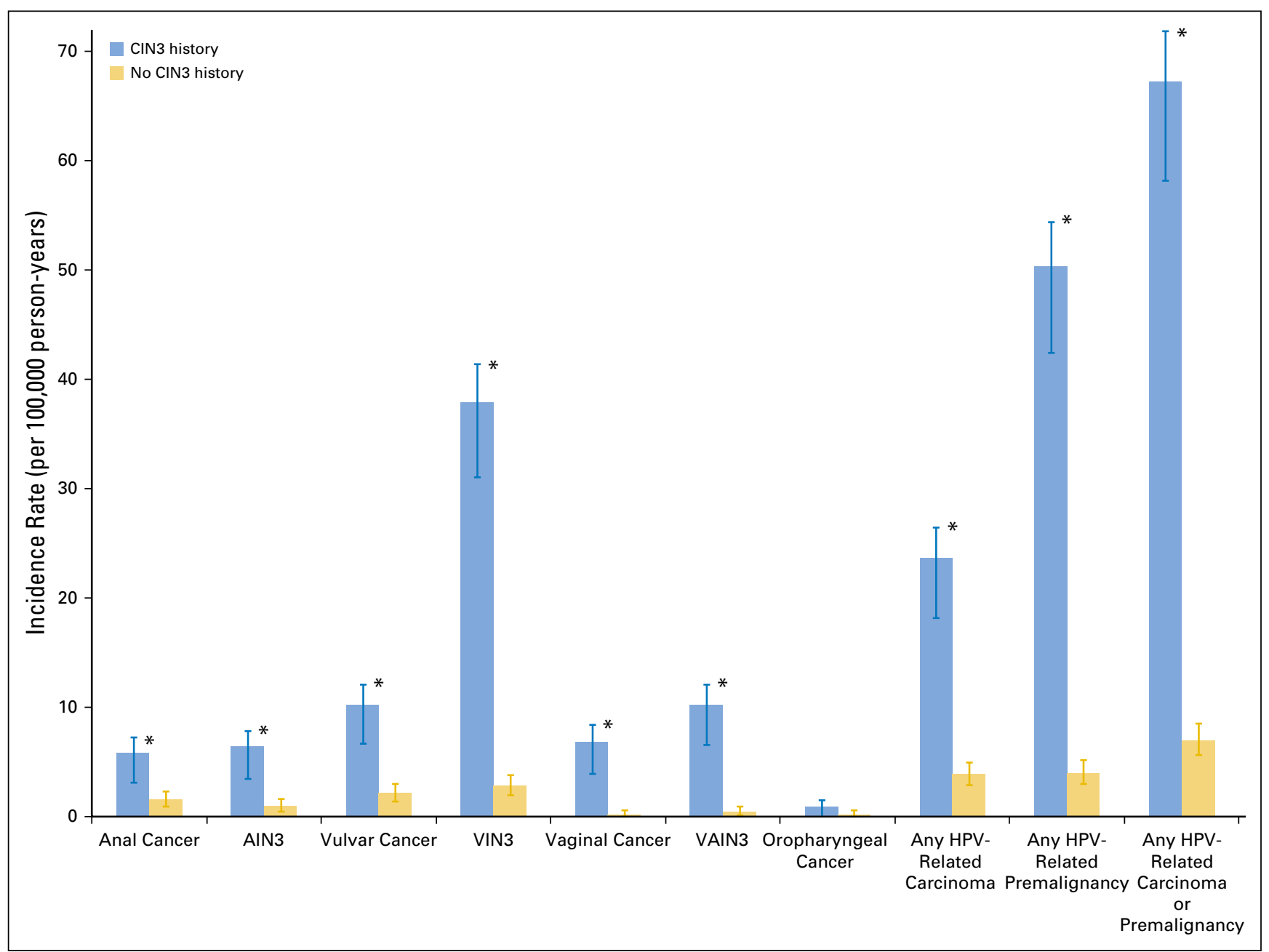

Fig 1. Estimated incidence rates of human papillomavirus (HPV)-related carcinomas and premalignancies, comparing women with and without a history of cervical

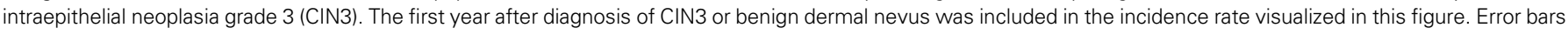

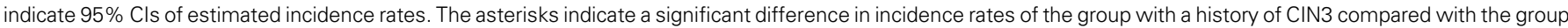

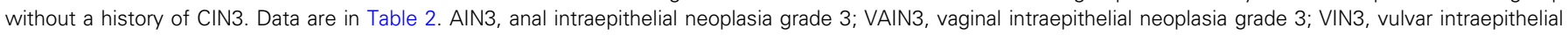
neoplasia grade 3.

Our results showed the highest IRRs for vaginal cancer and VAIN3 and the lowest IRR for anal cancer, which was still 3.85 (95\% CI, 2.32 to 6.37). Vaginal abnormalities are in proximity to cervical abnormalities; this makes a vaginal coinfection, with development of a vaginal carcinoma or premalignancy, more likely in women with a CIN3. This risk is much lower in women with no history of CIN3, which was shown by a low IR in the control group, resulting in a high IRR in vaginal cancer and VAIN3 after a CIN3. Also, in vulvar carcinomas and premalignancies, a loco-regional effect of HPV may explain an increased incidence of these lesions in women diagnosed with a CIN3. Women diagnosed with a CIN3 are commonly followed up with cervical smears or colposcopic examination. This may also result in increased detection of premalignancies of the vagina and vulva, with a higher IR in women with a CIN3 compared with women without a CIN3; this could result in an overestimation of IRR, especially in these two premalignancies. Also, not all anal, vulvar, vaginal, and oropharyngeal carcinomas and premalignancies are related to HPV; therefore, not all malignancies and premalignancies detected during follow-up in this study are related to HPV. We attempted to exclude carcinomas and premalignancies that were definitely not related to HPV. However, in the majority of cases, HPV tests were not performed on the histology samples; thus, some carcinomas and premalignancies not caused by HPV still would have been included. However, these carcinomas and premalignancies have been included in both the CIN3 group and in the control group of women without CIN3, with an expected similar number of cases; therefore, in estimated IRRs, these numbers cancel each other out. The overall increased IRR might also be explained by a woman's inability or limited ability to clear an hrHPV infection. A limited ability or inability to clear hrHPV infections did result in a persistent hrHPV infection with development of a CIN3 lesion, but could have also resulted in other HPV-related carcinomas and premalignancies. The long-lasting increased risk might also be explained by increased susceptibility to new hrHPV infections and recrudescence of disease because of an inability to clear the infection. It is known that smoking and immunodeficiency increases the risk of persistent hrHPV 


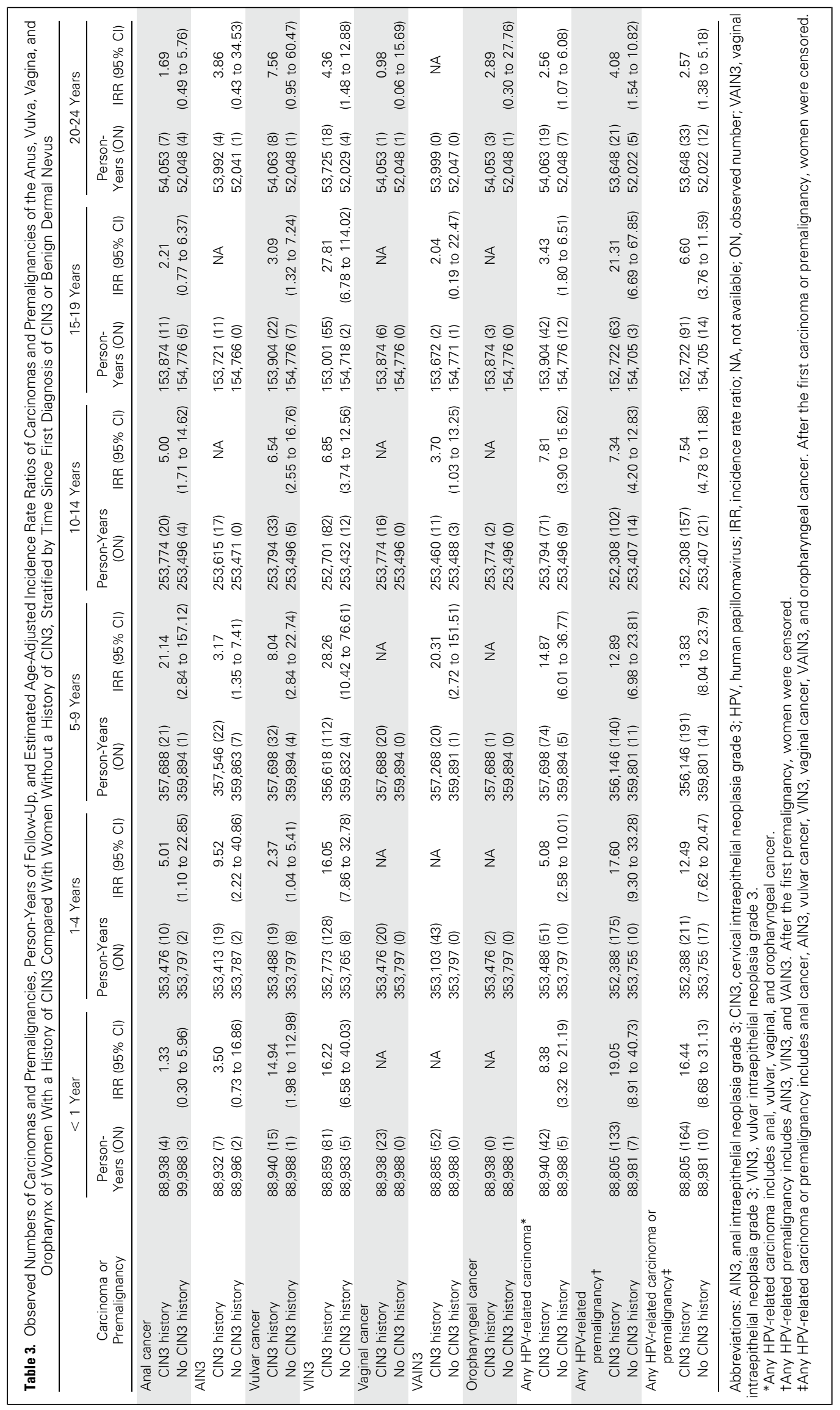




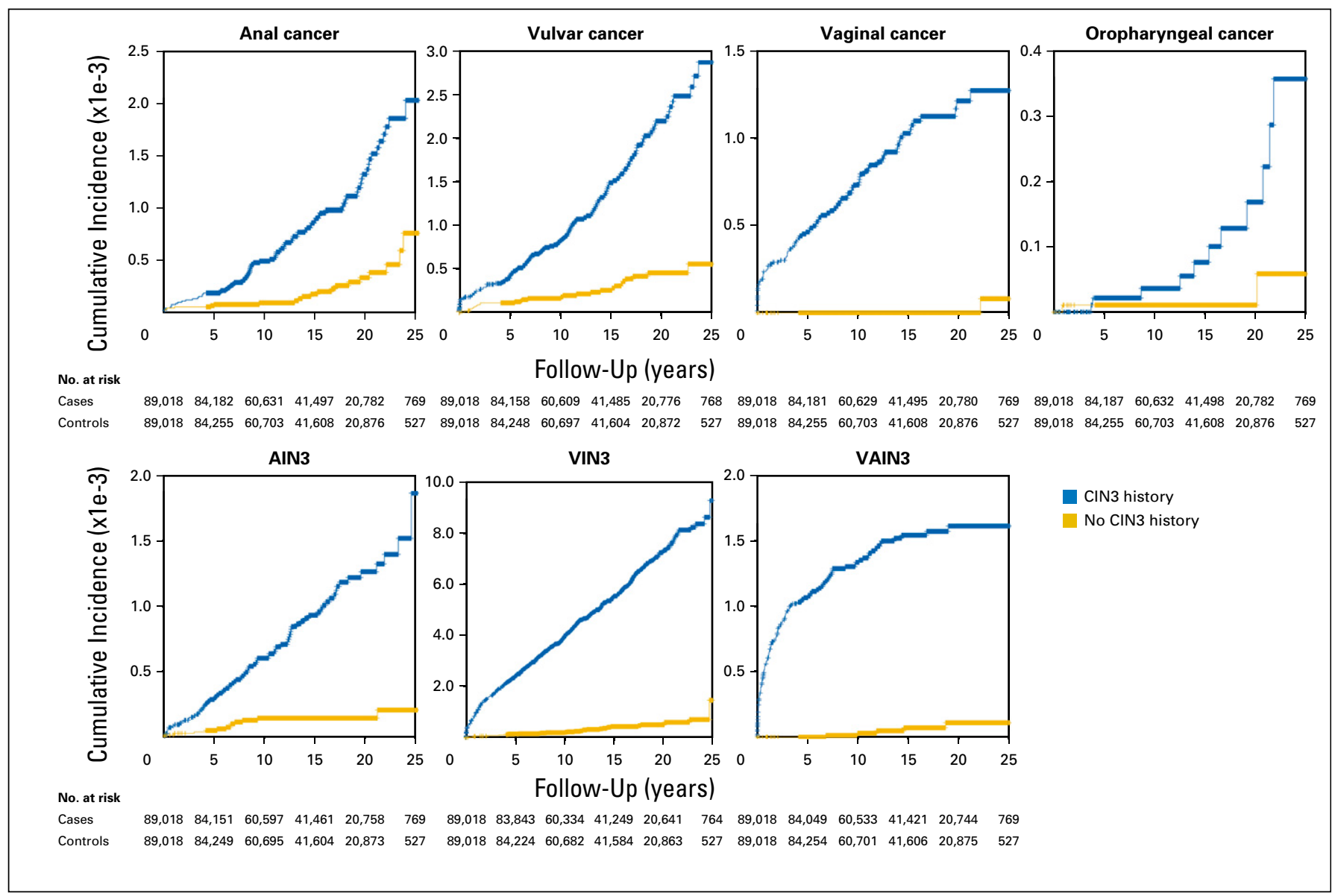

Fig 2. Kaplan-Meier curves estimating the cumulative incidence of human papillomavirus-related carcinomas and premalignancies after diagnosis of $\mathrm{CIN} 3$ or benign dermal nevus, comparing women with a previous diagnosis of CIN3 with women without CIN3. Note that the $y$-axis scale is not the same in all graphs. AIN3, anal intraepithelial neoplasia grade 3 ; CIN3, cervical intraepithelial neoplasia grade 3; VAIN3, vaginal intraepithelial neoplasia grade 3; VIN3, vulvar intraepithelial neoplasia grade 3.

infections, with an increased risk of (pre)malignant development. ${ }^{14-16}$ Data on smoking or immune status were not registered in the PALGA database, and could therefore not be

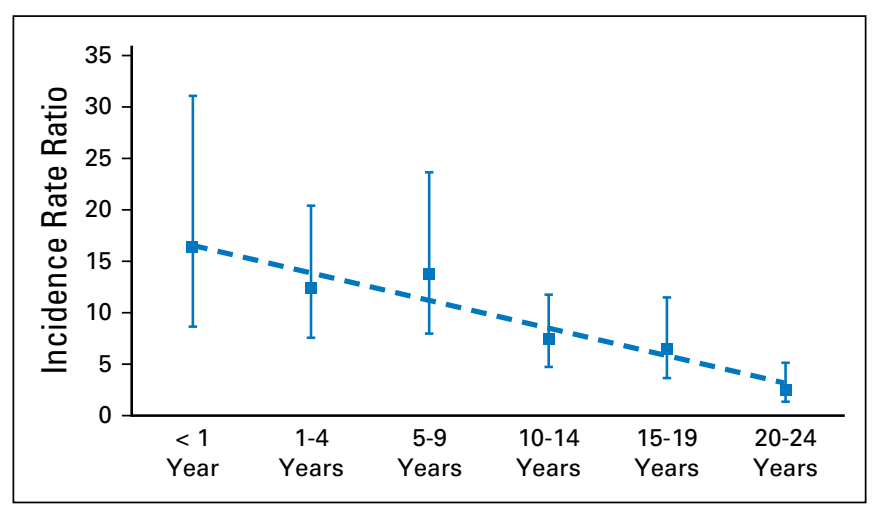

Fig 3. Incidence rate ratio of any human papillomavirus (HPV)-related carcinoma or premalignancy comparing women with a previous diagnosis of cervical intraepithelial neoplasia grade 3 with women without cervical intraepithelial neoplasia grade 3 , versus time. Any HPV-related carcinoma or premalignancy includes anal cancer, anal intraepithelial neoplasia grade 3, vulvar cancer, vulvar intraepithelial neoplasia grade 3, vaginal cancer, vaginal intraepithelial neoplasia grade 3 , and oropharyngeal cancer. Squares represent the incidence rate ratio at certain follow-up points. Error bars indicate 95\% Cls of estimated incidence rates. The dotted line is provided on the basis of simple linear regression for visualization purposes. Data are in Table 3. included in our analysis. Published results, however, show no increased risk explained by smoking. ${ }^{9}$

When looking at IRR over time, almost all IRRs showed a steady decline of increased risk during the long-term follow-up, with the lowest increased risk after 20 to 24 years. The KaplanMeier curves of all HPV-related carcinomas and premalignancies also confirmed this long-term increased risk. The increased risk of the cluster of any HPV-related carcinoma or premalignancy over time showed a decrease over time with an IRR of 16.44 (95\% CI, 8.68 to 31.13 ) in the first year, and an IRR of 2.57 (95\% CI, 1.38 to 5.18 ) after 20 years. This indicates that although the risk of HPVrelated carcinomas and premalignancies decreased over time, it was still significantly increased up to 20 years after the CIN3 diagnosis. A previous study by Gaudet et $\mathrm{al}^{8}$ also showed dropping incidences of malignancies over time, but to the contrary, Kalliala et $\mathrm{al}^{4}$ showed an increased standardized IR for malignancies over time after CIN3 treatment. For clusters of all carcinomas and/or all premalignancies, we also observed decreasing IRRs with advancing age. These data should be interpreted with some caution because the effect of a low incidence of malignancies in younger age groups might also be a confounding factor.

Ideally, we want to prevent the development of HPV-related carcinomas or high-grade premalignancies, or at least discover premalignancies before they progress to cancer. Higher alertness 
for HPV-related lesions in women with CIN3 might therefore be advisable. During colposcopy, it is relatively easy to look for vaginal carcinoma and premalignancies. Current guidelines advise to also examine the vaginal walls during colposcopic examination, and when an abnormal smear is not explained by cervical abnormalities, a vaginoscopy can be considered. When VAIN is diagnosed, it can be treated with surgical excision, laser evaporation, or imiquimod; however, recurrent disease is a known problem. ${ }^{17}$ Also, screening for vulvar and anal carcinoma and premalignancies might be easily combined with cervical diagnostics. Visual inspection will be easy to implement, but presently data are lacking that screening of these areas results in a lower cancer incidence. Indeed, there are no current screening programs for VIN, AIN, or oropharyngeal lesions, but if premalignancies are encountered, they are treated. ${ }^{18,19}$

Because an infection with hrHPV is causal to the development of other HPV-related carcinomas and high-grade premalignancies, prophylactic HPV vaccination may have a preventive effect on development of these lesions. However, the role of prophylactic vaccination in adult women is debated. Previous vaccination trials have shown that noninfected adults could potentially benefit from HPV vaccination. ${ }^{20,21}$ Garland et $\mathrm{al}^{22}$ showed that women undergoing surgical therapy for cervical lesions after vaccination with the bivalent vaccine may benefit from vaccination, with a reduced risk of developing subsequent CIN2 positivity. Studies in women with a current infection with hrHPV showed varying results. Two recent studies showed a reduced incidence of subsequent HPVrelated disease in women vaccinated with the quadrivalent HPV vaccine after surgical treatment of CIN3 when compared with surgically treated women who did not receive the HPV vaccination. ${ }^{23,24}$ On the contrary, a recent study by Hildesheim et $\mathrm{al}^{25}$ showed no evidence for a vaccine effect on detectable HPV infections in hrHPV-positive women and women undergoing treatment of cervical premalignancy. Also, the small potential harms of vaccination should be taken into account, alongside the fact that HPV infections leading to premalignancies or malignancies may have been acquired before the HPV vaccination. It is therefore not fully clarified how clinically and cost effective prophylactic hrHPV vaccination would be in women treated for CIN3. Randomized controlled trials and cost-effectiveness studies might answer these questions. Our study, however, showed that shortand long-term risks of HPV-related abnormalities are strongly increased in women diagnosed with CIN3. Patients should be informed on an individual basis about this increased risk. The Dutch guideline presently advises to consider hrHPV vaccination in women diagnosed with CIN3, until more conclusive evidence on vaccine effect after treatment of CIN3 is available. ${ }^{17}$

The introduction of prophylactic HPV vaccination is expected to reduce the incidence of HPV-related cancers; postvaccination surveillance studies have supported this by showing a significant reduction of $68 \%$ in type 16 and 18 infections in countries with female vaccination coverage of $\geq 50 \% .^{26-29}$ However, monitoring remains essential to identify possible waning of efficacy or replacement of HPV type. Because vaccination programs for young women only started in 2007, it will take many more years before the effects of HPV vaccination become visible in the total adult female population.

In conclusion, this population-based data set shows further evidence of the increased risk of HPV-related carcinomas and premalignancies of the anogenital and oropharyngeal regions after a diagnosis of CIN3, and gives a clear view of women at risk for HPV-related disease. Studies that investigate methods to prevent this increased risk in this group of patients, such as intensified screening or vaccination, are warranted.

\section{AUTHORS' DISCLOSURES OF POTENTIAL CONFLICTS OF INTEREST}

Disclosures provided by the authors are available with this article at jco.org.

\section{AUTHOR CONTRIBUTIONS}

Conception and design: Renée M.F. Ebisch, Joanna IntHout, Willem J.G. Melchers, Ruud L.M. Bekkers, Albert G. Siebers

Collection and assembly of data: Renée M.F. Ebisch, Dominiek W.E. Rutten, Albert G. Siebers

Data analysis and interpretation: Renée M.F. Ebisch, Dominiek W.E. Rutten, Joanna IntHout, Leon F.A.G. Massuger, Johan Bulten, Ruud L.M. Bekkers, Albert G. Siebers

Manuscript writing: All authors

Final approval of manuscript: All authors

Accountable for all aspects of the work: All authors

\section{REFERENCES}

1. Parkin DM: The global health burden of infection-associated cancers in the year 2002. Int J Cancer 118:3030-3044, 2006

2. Parkin DM, Bray F: Chapter 2: The burden of HPV-related cancers. Vaccine 24(Suppl 3):S11-S25, 2006

3. de Martel C, Ferlay J, Franceschi S, et al: Global burden of cancers attributable to infections in 2008: A review and synthetic analysis. Lancet Oncol 13:607-615, 2012

4. Kalliala I, Anttila A, Pukkala E, et al: Risk of cervical and other cancers after treatment of cervical intraepithelial neoplasia: Retrospective cohort study. BMJ 331:1183-1185, 2005
5. Evans HS, Newnham A, Hodgson SV, et al: Second primary cancers after cervical intraepithelial neoplasia III and invasive cervical cancer in Southeast England. Gynecol Oncol 90:131-136, 2003

6. Gillison ML, Chaturvedi AK, Lowy DR: HPV prophylactic vaccines and the potential prevention of noncervical cancers in both men and women. Cancer 113(10 Suppl)3036-3046, 2008

7. Strander B, Andersson-Ellström A, Milsom I, et al: Long term risk of invasive cancer after treatment for cervical intraepithelial neoplasia grade 3: Population based cohort study. BMJ 335:1077, 2007

8. Gaudet M, Hamm J, Aquino-Parsons C: Incidence of ano-genital and head and neck malignancies in women with a previous diagnosis of cervical intraepithelial neoplasia. Gynecol Oncol 134: 523-526, 2014
9. Edgren G, Sparén P: Risk of anogenital cancer after diagnosis of cervical intraepithelial neoplasia: A prospective population-based study. Lancet Oncol 8: 311-316, 2007

10. Scholefield JH, Sonnex C, Talbot IC, et al: Anal and cervical intraepithelial neoplasia: Possible parallel. Lancet 2:765-769, 1989

11. Pirog EC, Kleter B, Olgac S, et al: Prevalence of human papillomavirus DNA in different histological subtypes of cervical adenocarcinoma. Am J Pathol 157:1055-1062, 2000

12. van der Avoort IA, Shirango $H$, Hoevenaars BM, et al: Vulvar squamous cell carcinoma is a multifactorial disease following two separate and independent pathways. Int J Gynecol Pathol 25:22-29, 2006

13. Insinga $R P$, Liaw $K L$, Johnson $L G$, et al: $A$ systematic review of the prevalence and attribution 
of human papillomavirus types among cervical, vaginal, and vulvar precancers and cancers in the United States. Cancer Epidemiol Biomarkers Prev 17: 1611-1622, 2008

14. Appleby $P$, Beral V, Berrington de González $A$ et al: Carcinoma of the cervix and tobacco smoking: Collaborative reanalysis of individual data on 13,541 women with carcinoma of the cervix and 23,017 women without carcinoma of the cervix from 23 epidemiological studies. Int J Cancer 118:1481-1495, 2006

15. Vaccarella S, Herrero R, Snijders PJ, et al: Smoking and human papillomavirus infection: Pooled analysis of the International Agency for Research on Cancer HPV Prevalence Surveys. Int J Epidemiol 37: 536-546, 2008

16. Grulich $A E$, van Leeuwen $M T$, Falster $M O$, et al: Incidence of cancers in people with HIV/AIDS compared with immunosuppressed transplant recipients: A meta-analysis. Lancet 370:59-67, 2007

17. Integraal Kankercentrum Nederland, Werk groep Richtlijn CIN, AIS en VAIN: Dutch Nationwide Guideline CIN, AIS and VAIN, version 1.0. 12-11 2015. http://www.oncoline.nl/cin-ais-en-vain

18. Integraal Kankercentrum Nederland, Werkgroep Oncologische Gynaecologie: Dutch Nationwide Guideline Vulvar Intraepithelial Neoplasia (VIN) version 1.3. 08-12-2010. http://www.oncoline.nl/vin
19. Integraal Kankercentrum Nederland, Werkgroep Gastro-Intestinale Tumoren: Dutch Nationwide Guideline Anal Cancer, version 2.0. 13-11-2012. http://www oncoline.nl/anuscarcinoom

20. Muñoz N, Manalastas R Jr, Pitisuttithum $P$, et al: Safety, immunogenicity, and efficacy of quadrivalent human papillomavirus (types $6,11,16,18$ ) recombinant vaccine in women aged $24-45$ years: $A$ randomised, double-blind trial. Lancet 373: 1949-1957, 2009

21. Schwarz TF, Spaczynski M, Schneider A, et al: Immunogenicity and tolerability of an HPV-16/18 AS04-adjuvanted prophylactic cervical cancer vac cine in women aged $15-55$ years. Vaccine 27 581-587, 2009

22. Garland SM, Paavonen J, Jaisamrarn U, et al: Prior human papillomavirus-16/18 AS04-adjuvanted vaccination prevents recurrent high grade cervical intraepithelial neoplasia after definitive surgical therapy: Post-hoc analysis from a randomized controlled trial. Int J Cancer 139:2812-2826, 2016

23. Kang WD, Choi HS, Kim SM: Is vaccination with quadrivalent HPV vaccine after loop electrosurgical excision procedure effective in preventing recurrence in patients with high-grade cervical intraepithelial neoplasia (CIN2-3)? Gynecol Oncol 130:264-268, 2013
24. Joura EA, Garland SM, Paavonen J, et al: Effect of the human papillomavirus (HPV) quadrivalent vaccine in a subgroup of women with cervical and vulvar disease: Retrospective pooled analysis of trial data. BMJ 344:e1401, 2012

25. Hildesheim A, Gonzalez P, Kreimer AR, et al: Impact of human papillomavirus (HPV) 16 and 18 vaccination on prevalent infections and rates of cervical lesions after excisional treatment. Am J Obstet Gynecol 215:212.e1-212.e15, 2016

26. Jit $M$, Brisson $M$, Portnoy $A$, et al: Costeffectiveness of female human papillomavirus vaccination in 179 countries: A PRIME modelling study. Lancet Glob Health 2:e406-e414, 2014

27. Brisson M, Van de Velde N, Boily MC: Economic evaluation of human papillomavirus vaccination in developed countries. Public Health Genomics 12:343-351, 2009

28. FUTURE II Study Group: Quadrivalent vaccine against human papillomavirus to prevent high-grade cervical lesions. N Engl J Med 356: 1915-1927, 2007

29. Drolet M, Bénard É, Boily $M C$, et al: Population-level impact and herd effects following human papillomavirus vaccination programmes: A systematic review and meta-analysis. Lancet Infect Dis 15:565-580, 2015

\section{Affiliations}

Renée M.F. Ebisch, Dominiek W.E. Rutten, Joanna IntHout, Willem J.G. Melchers, Leon F.A.G. Massuger, Johan Bulten, Ruud L.M. Bekkers, and Albert G. Siebers, Radboud University Medical Center, Nijmegen; and Ruud L.M. Bekkers, Catharina Hospital Eindhoven, Eindhoven, the Netherlands. 


\section{AUTHORS' DISCLOSURES OF POTENTIAL CONFLICTS OF INTEREST}

Long-Lasting Increased Risk of Human Papillomavirus-Related Carcinomas and Premalignancies After Cervical Intraepithelial Neoplasia Grade 3: A Population-Based Cohort Study

The following represents disclosure information provided by authors of this manuscript. All relationships are considered compensated. Relationships are self-held unless noted. I = Immediate Family Member, Inst = My Institution. Relationships may not relate to the subject matter of this manuscript. For more information about ASCO's conflict of interest policy, please refer to www.asco.org/rwc or ascopubs.org/jco/site/ifc.

Renée M.F. Ebisch

No relationship to disclose

\section{Dominiek W.E. Rutten}

No relationship to disclose

\section{Joanna IntHout}

No relationship to disclose

Willem J.G. Melchers

No relationship to disclose

\section{Leon F.A.G. Massuger}

No relationship to disclose

Johan Bulten

No relationship to disclose

Ruud L.M. Bekkers

Research Funding: Roche Diagnostics (Inst), Merck Sharp \& Dohme (Inst)

Albert G. Siebers

No relationship to disclose 


\section{Appendix}

\begin{tabular}{|c|c|c|c|c|c|c|c|c|}
\hline $\begin{array}{l}\text { Carcinoma or } \\
\text { Premalignancy }\end{array}$ & \multicolumn{2}{|c|}{$\leq 29$ Years } & \multicolumn{2}{|c|}{ 30-49 Years } & \multicolumn{2}{|c|}{ 50-69 Years } & \multicolumn{2}{|c|}{$\geq 70$ Years } \\
\hline \multicolumn{9}{|c|}{ Any HPV-related carcinoma* } \\
\hline CIN3 history & $\begin{array}{l}34,739(4) \\
42,885(0)\end{array}$ & NA & $\begin{array}{l}836,486(133) \\
813687(16)\end{array}$ & $\begin{array}{c}8.09 \\
(481+01358)\end{array}$ & $\begin{array}{l}355,154(123) \\
370,017(27)\end{array}$ & $\begin{array}{c}4.75 \\
(3.13+0720)\end{array}$ & $\begin{array}{l}38,796(39) \\
39,931(5)\end{array}$ & $\begin{array}{c}8.03 \\
(316+02037)\end{array}$ \\
\hline \multicolumn{9}{|l|}{$\begin{array}{l}\text { Any HPV-related } \\
\text { premalignancy } \dagger\end{array}$} \\
\hline $\begin{array}{l}\text { CIN3 history } \\
\text { No CIN3 history }\end{array}$ & $\begin{array}{l}34,616(29) \\
42,884(1)\end{array}$ & $\begin{array}{c}35.93 \\
(4.89 \text { to } 263.72)\end{array}$ & $\begin{array}{l}833,203(427) \\
813,557(25)\end{array}$ & $\begin{array}{c}16.68 \\
(11.14 \text { to } 24.96)\end{array}$ & $\begin{array}{l}353,158(150) \\
369,847(21)\end{array}$ & $\begin{array}{c}7.48 \\
(4.74 \text { to } 11.81)\end{array}$ & $\begin{array}{l}38,329(28) \\
39,905(3)\end{array}$ & $\begin{array}{c}9.72 \\
\text { (2.95 to } 31.96)\end{array}$ \\
\hline \multicolumn{9}{|c|}{$\begin{array}{l}\text { Any HPV-related carcinoma } \\
\text { or premalignancy } \neq\end{array}$} \\
\hline $\begin{array}{l}\text { CIN3 history } \\
\text { No CIN3 history }\end{array}$ & $\begin{array}{l}34,616(32) \\
42,884(1)\end{array}$ & $\begin{array}{c}39.64 \\
\text { (5.42 to } 290.09)\end{array}$ & $\begin{array}{l}833,203(516) \\
813,557(37)\end{array}$ & $\begin{array}{c}13.62 \\
\text { (9.76 to } 19.01)\end{array}$ & $\begin{array}{l}353,158(245) \\
369,847(45)\end{array}$ & $\begin{array}{c}5.70 \\
\text { (4.15 to } 7.84)\end{array}$ & $\begin{array}{l}38,329(54) \\
39,905(5)\end{array}$ & $\begin{array}{c}11.24 \\
\text { (4.50 to } 28.11)\end{array}$ \\
\hline
\end{tabular}

